\title{
Atmospheric monitoring with the Fluorescence detector Array of Single-pixel Telescopes
}

\author{
Dusan Mandat ${ }^{1,5, *}$, Toshihiro Fujii ${ }^{2}$, Max Malacari ${ }^{3}$, John Farmer $^{3}$, Xiaochen $\mathrm{Ni}^{3}$, Paolo Privitera ${ }^{3}$, Justin Albury ${ }^{4}$, Jose \\ A. Bellido ${ }^{4}$, Petr $\mathrm{Hamal}^{5}$, Pavel Horvath ${ }^{5}$, Miroslav Hrabovsky ${ }^{5}$, Libor Nozka ${ }^{1,5}$, Ladislav Chytka ${ }^{1,5}$, Miroslav Palatka ${ }^{1,5}$, \\ Miroslav Pech ${ }^{1,5}$, Petr Schovanek ${ }^{1,5}$, Petr Travnicek ${ }^{1,5}$, John N. Matthews ${ }^{6}$, and Stan Thomas ${ }^{6}$ \\ ${ }^{1}$ Institute of Physics of the Academy of Sciences of the Czech Republic, Prague, Czech Republic \\ ${ }^{2}$ Institute for Cosmic Ray Research, University of Tokyo, Kashiwa, Chiba, Japan \\ ${ }^{3}$ Kavli Institute for Cosmological Physics, University of Chicago, Chicago, IL, USA \\ ${ }^{4}$ Department of Physics, University of Adelaide, Adelaide, S.A., Australia \\ ${ }^{5}$ Palacky University, RCPTM, Olomouc, Czech Republic \\ ${ }^{6}$ High Energy Astrophysics Institute and Department of Physics and Astronomy, University of Utah, Salt Lake City, UT, USA
}

\begin{abstract}
The Fluorescence detector Array of Single-pixel Telescopes (FAST) is a proposed low-cost, largearea, next-generation experiment for the detection of ultrahigh-energy cosmic rays (UHECRs) via the atmospheric fluorescence technique. Two FAST telescopes are currently installed and operating at the Black Rock Mesa site of the Telescope Array Experiment in Utah, USA. Knowledge of the properties of the atmosphere above the detector is of utmost importance for the analysis and reconstruction of the energy and trajectory of UHECRs measured with an atmospheric fluorescence telescope. The FAST experiment uses all sky camera (FASCam) and sky quality monitor (SQM) for the detection of clouds and quantification of the night-sky background light in the field-of-view of the telescopes. Measurements of a vertically-fired ultra-violet laser at a distance of $21 \mathrm{~km}$ from the FAST telescopes are used to infer the transparency of the atmosphere above the detector through comparison with simulations.
\end{abstract}

\section{Introduction}

The Fluorescence detector Array of Single-pixel Telescopes (FAST) [1] is a design concept for a low-cost, ground-based fluorescence detector (FD). A FAST telescope consists of just four pixels covering a $30^{\circ} \times 30^{\circ}$ patch of the sky with a $\sim 1 \mathrm{~m}^{2}$ collecting area. Its low cost will facilitate deployment over a very large ground area, making it a viable candidate for a next-generation cosmic ray observatory.

In October 2016, the first full-scale FAST prototype was installed in a dedicated building at the Telescope Array site in central Utah, USA, with a second telescope following in an adjacent building in September 2017. Both telescopes are fully remotely-operable, and record data in coincidence with the adjacent Black Rock Mesa FD site. Collected data include measurements of air showers, as well as vertical laser traces from the Telescope Array's Central Laser Facility (CLF).

\section{Atmospheric monitoring devices}

The FAST telescope is located at the Telescope Array's Black Rock Mesa site. A number of atmospheric monitoring devices are in place to collect data useful in the

*e-mail: mandat@fzu.cz analysis of air shower measurements. The TA fluorescence detector building is equipped with several weather instruments, including a weather station, and wind and rain sensors. Due to their close proximity $(<100 \mathrm{~m})$ to the FAST prototype telescopes, data from these instruments are made available to the FAST collaboration for use in air shower and laser analyses. The FAST all sky camera (FASCam) and sky quality monitor (SQM) are two instruments attached to the first FAST telescope building for monitoring of the night-sky background and cloud coverage. The Central Laser Facility, located at a distance of $21 \mathrm{~km}$ from the Black Rock Mesa site, fires a series of 300 vertical laser shots through the field of view of one FAST telescope every half hour, and measurements of this signal serve as an important monitoring tool for the transparency of the atmosphere.

\subsection{FAST all sky camera (FASCam)}

The FASCam is a fully automated all sky camera designed to measure the cloud coverage above the detector. FASCam has a $\sim 180^{\circ}$ field of view and is equipped with a series of Johnson filters, as well as a UV filter. The camera is installed on the top of one of the FAST telescope buildings together with the SQM (see Fig. 1). FASCam provides $30 \mathrm{~s}$ exposures of the night sky using the Johnson filters, as well as a $180 \mathrm{~s}$ exposure through the UV 


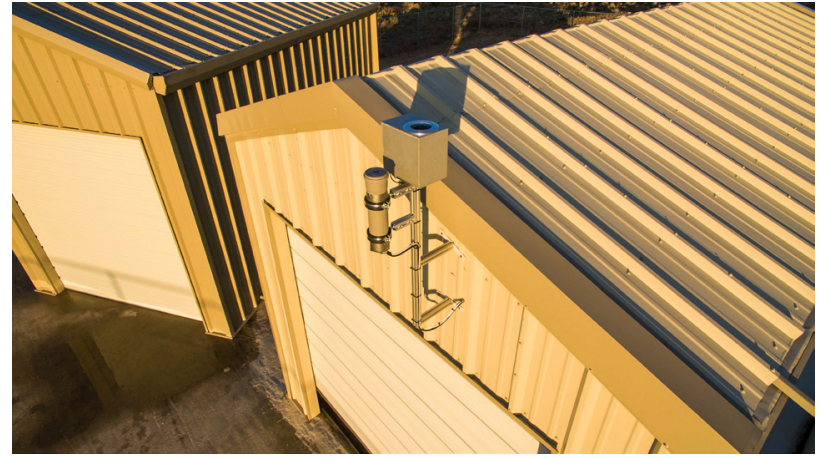

Figure 1. The FASCam and SQM installed on the roof of one of the FAST telescope buildings.

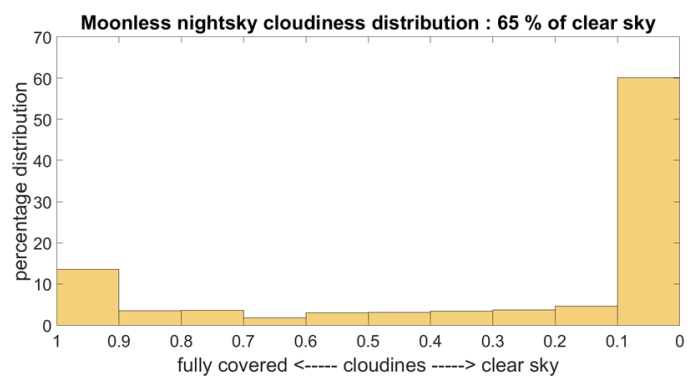

Figure 2. Cloudiness of the FAST site. The visible star fraction (ratio of visible stars to observable catalog stars) defines the cloudiness. A cloudiness of 0 corresponds to a cloud-free sky, while a cloudiness of 1 corresponds to an overcast sky.

filter. An astrometry-based cloud analysis compares images of star positions with a catalog of known coordinates in each wavelength band, and calculates the ratio of the number of visible to observable stars in order to estimate the could coverage. The setup is similar to that of the CTA All Sky Camera [2] and the Pierre Auger Observatory's Background Camera [3]. Measurements taken since the FASCam was installed in September 2017 suggest a clear sky during $65 \%$ of the measurement time (a clear sky is defined as a visible/observable fraction $>0.8$ ). A histogram of the cloudiness over the past year is shown in Fig. 2. Images taken with the UV filter can be used to characterise the night-sky background within the field of view of individual FAST PMTs (in the case that the sky is free of clouds).

The cloud coverage is continuously monitored during FAST data-taking, and is available to shift operators through a simple web-based monitoring interface. In the case of very cloudy conditions, data-taking can be paused until conditions improve (see Fig. 3).

\subsection{Sky quality monitor (SQM)}

The SQM is a commercial device used to measure the night-sky brightness (NSB) in magnitudes per square arcsecond (see Fig. 1). The device has a precision of $\pm 0.1 \mathrm{mag} / \operatorname{arcsec}^{2}$. Measurements taken over the past year suggest a median NSB of $21.6 \mathrm{mag} / \mathrm{arcsec}^{2}$, similar to that of the CTA candidate sites [4].

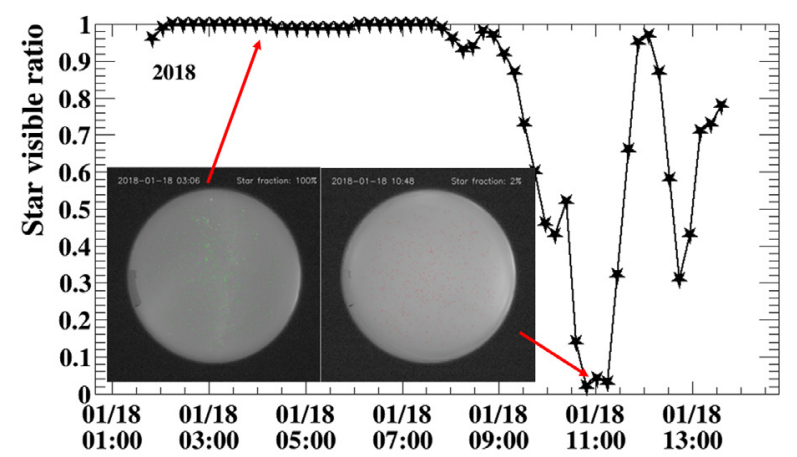

Figure 3. The clouds are continuously monitored during FAST operation. In the case of an overcast sky data acquisition is terminated, and the operator waits until conditions improve.

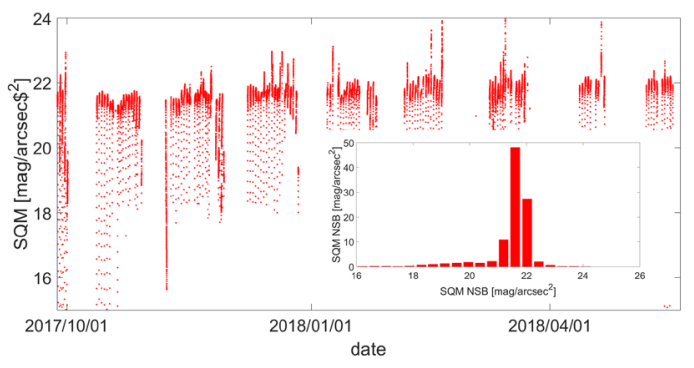

Figure 4. The night-sky background measured using the SQM. The inset shows a histogram of the NSB. The median of the data set is $21.6 \mathrm{mag} / \mathrm{arcsec}^{2}$.

\section{Measurement of the night-sky background using the FAST telescopes}

The average photocathode current $I_{\mathrm{pe}}$ of the PMTs in a FAST telescope is dominated by the night-sky background. The NSB is caused primarily by bright stars within the telescope field-of-view, and other natural or artificial light sources. AC coupling of the FAST PMTs does not allow for a direct measurement of the average photocathode current. However, fluctuations in the NSB are recorded as a fluctuations of the PMT pedestal, whose variance is linearly related to the average current [5].

Two types of pedestal measurements are recorded, first with the shutter closed at the beginning of an observing run, and then with the shutter opened at 5 minute intervals during data-taking. With the shutter closed the pedestal fluctuations are dominated by the FAST electronics noise, generating $\sim 13$ p.e. $/ 20 \mathrm{~ns}$. With the shutter opened the measured photocathode current of $\sim 98$ p.e. $/ 20 \mathrm{~ns}$ is dominated by the NSB, indicating that the electronic noise is negligible with respect to the NSB. The evolution of the NSB during a clear-night run of continuous data-taking is shown in Fig. 5. 


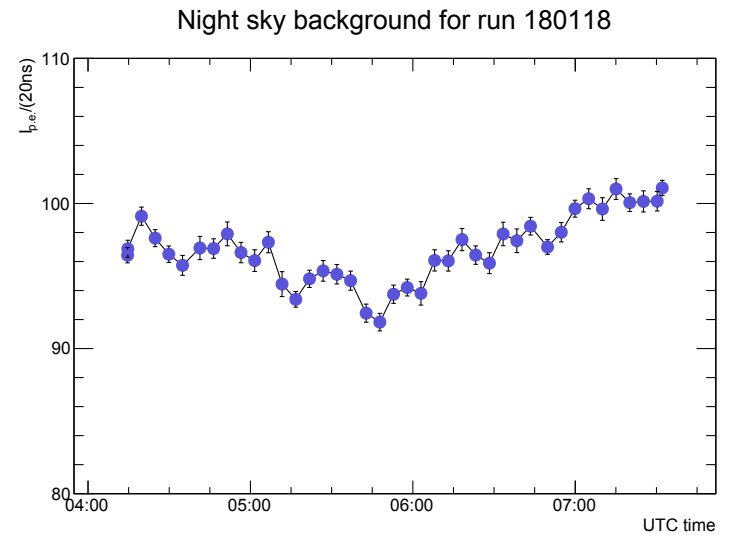

Figure 5. The evolution of the photocathode current during a clear-night run.

\section{Measurement of the night-sky background using the FASCam}

Knowledge of the night-sky background before and during data-taking is very important. The NSB decreases after sunset, and must monitored to determine the time at which it is safe to open the telescope shutter and begin data acquisition. The NSB can also fluctuate throughout the night, and can increase as a result of both artificial (such as car headlights, aeroplanes, and light pollution) and natural (eg. lightning) light sources, as well as being affected by cloud coverage and atmospheric transparency (eg. aerosols). The FASCam can be used for monitoring of the NSB in the field of view of the FAST telescopes. As the pointing direction of each pixel of FASCam is calculated using photometry, we are able to map the FASCam pixels onto the FAST PMTs, and hence estimate the NSB signal from the sky exposure in the UV band (see Fig. 6). The signal is compared with the NSB from the PMTs (see Sec. 3 and Fig. 7) and shows good agreement with the data.

\section{Atmospheric characterisation using the Central Laser Facility}

The faint fluorescence light produced during the development of an air shower is attenuated on its way to a FAST telescope due to elastic molecular (Rayleigh) and aerosol (Mie) scattering. In addition, strongly forward-beamed Cherenkov light produced by relativistic electrons in the shower can be scattered into the FAST field-of-view. For the highest energy cosmic rays, light from an air shower may have to travel up to $40 \mathrm{~km}$ from its point of emission to a FAST telescope, meaning that the transmission properties of the atmosphere must be very well understood.

FAST can be used as an atmospheric monitoring tool by observing vertical laser shots fired by the Telescope Array's Central Laser Facility (CLF)[6]. The TA CLF fires 300 vertical shots $(\sim 4.0 \mathrm{~mJ}$ at $355 \mathrm{~nm}, 10 \mathrm{~Hz}$, near the middle of the fluorescence wavelength range) every $30 \mathrm{~min}-$ utes during data taking for atmospheric monitoring purposes. The aerosol content of the atmosphere can be in-

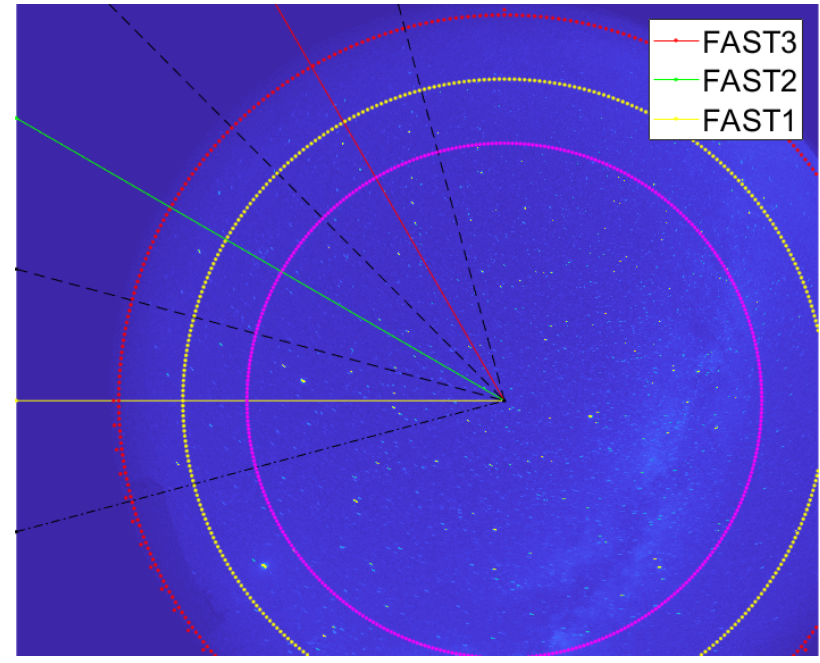

Figure 6. A FASCam night-sky image in the UV band. The sky can be split into individual zones corresponding to FAST pixels. Stars are visible in the FAST FOV. The integrated signal from the pixels corresponding to the FAST PMTs can be analysed and compared with the PMT NSB.

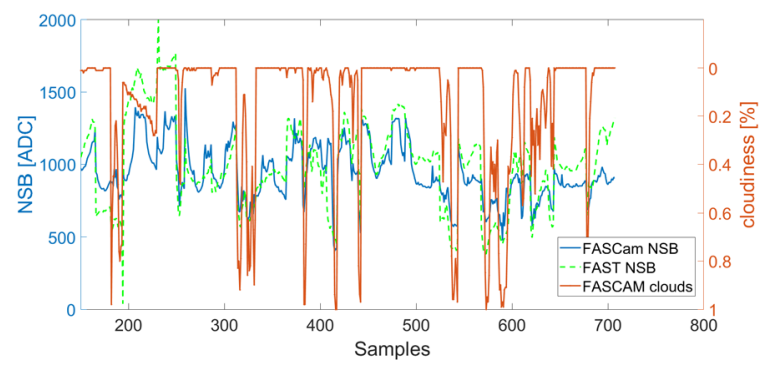

Figure 7. NSB comparison between FAST PMT data and FASCam images.

ferred from these measured laser traces through comparisons with simulations, and is typically expressed in terms of the vertical aerosol optical depth (VAOD), the integral of the aerosol extinction coefficient $\alpha$ from the ground to height $h$

$$
\operatorname{VAOD}(h)=\int_{0}^{h} \alpha\left(h^{\prime}\right) \mathrm{d} h^{\prime} .
$$

A preliminary comparison between a set of 250 laser shots measured during a data-taking run at the TA site, and simulations of the expected laser signal under varying aerosol attenuation conditions, is shown in Fig. 8. The simulation takes into account the wavelength-dependent attenuation of a laser beam as it traverses a parameterised atmosphere, as well as the attenuation of light scattered out of the beam towards the FAST telescope aperture. The expected signal is then calculated taking into account the measured optical properties of the FAST telescope installed at the Black Rock Mesa site, the typical night-sky background noise rate, and the laboratory-measured PMT response. Simulations are shown for a pure molecular atmosphere, and for VAODs (integrated to the top of the atmosphere) of 0.04 and 0.1 , consistent with typical and 


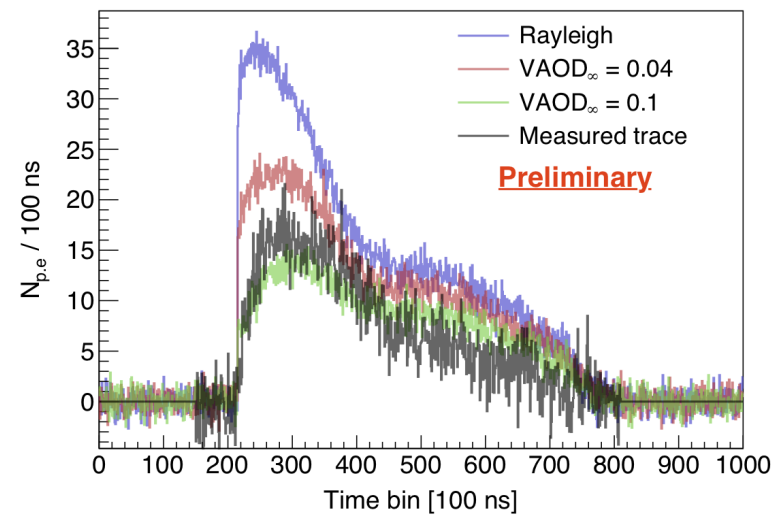

Figure 8. Average measured signal from 250 vertical CLF shots measured at the TA site, compared with the expectation from simulations for 3 different aerosol atmospheres. An aerosol scale height of $1 \mathrm{~km}$ was assumed for all atmospheres, consistent with the TA assumption.

high atmospheric aerosol loadings at the TA site. For the latter two cases the aerosol atmosphere was assumed to have a scale height of $1 \mathrm{~km}$. While this comparison is preliminary, it demonstrates FAST's excellent sensitivity to vertical laser shots and highlights the potential for FAST to contribute to a future ground-based observatory's atmospheric monitoring efforts.

\section{Conclusions}

The FAST telescopes installed at the Telescope Array site in Utah, USA, are supported by several important atmospheric devices, each collecting data useful for both online monitoring of environmental conditions, and for offline air shower and laser analyses. The online data are an indispensable tool, invaluable for shifters partaking in the remote operation of the telescopes. All weather and atmospheric data are available for further offline data analysis, and for long-term characterisation of the atmosphere above the detector.

\section{Acknowledgements}

This work was supported by the Japan Society for the Promotion of Science (JSPS) through the Grant-in-Aid for Scientific Research (B) 18H01225, the Grant-in-Aid for Young Scientist (A) 15H05443, Grant-in-Aid for JSPS Research Fellow 16J04564 and JSPS Fellowships H25-339, H28-4564. This work was partially carried out by the joint research program of the Institute for Cosmic Ray Research (ICRR), University of Tokyo. This work was supported in part by NSF grant PHY1713764, PHY-1412261 and by the Kavli Institute for Cosmological Physics at the University of Chicago through grant NSF PHY-1125897 and an endowment from the Kavli Foundation and its founder Fred Kavli. The Czech authors gratefully acknowledge the support of the Ministry of Education, Youth and Sports of the Czech Republic projects CZ.02.1.01/0.0/0.0/16_013/0001402, CZ.02.1.01/0.0/0.0/17_049/0008422, LTAUSA17078, LTT18004.

\section{References}

[1] T. Fujii et al., Detection of ultra-high energy cosmic ray showers with a single-pixel fluorescence telescope, Astropart. Phys., 74, 64-72, (2015) [1504.00692], https://doi.org/10.1016/j.astropartphys.2015.10.006

[2] D. Mandat, M.Pech, All Sky Camera for CTA Site characterization, EPJ Web Conf., 144, 1-3 (2017), https://doi.org/10.1051/epjconf/201714401005

[3] D. Mandat, et al. All Sky Camera instrument for night sky monitoring, Proceedings of the Atmospheric Monitoring for High-Energy Astroparticle Detectors (AtmoHEAD) Conference, 1-4 (2014), arXiv:1402.4762 [astro-ph.IM]

[4] Ch. Fruk et al., Instrumentation for comparing night sky quality and atmospheric conditions of CTA site candidates, Journal of Instrumentation, 10, (2015) [10.1088/1748-0221/10/04/P04012]

[5] H. Gemmeke, M. Kleifges, and A. Menshikov, Statistical calibration and background measurements of the Auger fluorescence detector, Forschungszentrum Karlsruhe Report FZKA (2003) 891-894

[6] S. Udo et al., The Central Laser Facility at the Telescope Array, Proc. of the 30th International Cosmic Ray Conference, Merida, Mexico 5 (2007) 1021-1024. 